\title{
Torsades de pointes in a patient of dilated cardiomyopathy occurring early on oral amiodarone therapy
}

\author{
Mohit D. Gupta*, Girish MP, Partha Prateem Choudhury, Sanjay Tyagi
}

\begin{abstract}
Department of Cardiology, GB Pant Hospital and associated Maulana Azad Medical College, New Delhi, India
\end{abstract}

Received: 20 June 2013

Accepted: 28 June 2013

\section{*Correspondence to: \\ Dr. Mohit D. Gupta, \\ Email: drmohitgupta@yahoo.com}

(C) 2013 Gupta MD et al. This is an open-access article distributed under the terms of the Creative Commons Attribution License, which permits unrestricted use, distribution, and reproduction in any medium, provided the original work is properly cited.

\begin{abstract}
Amiodarone induced proarrhythmic effects are rare. We report a case of amiodarone induced torsades de pointes in a young boy aged 16 years occurring early after initiation of oral amiodarone. This case underscores the need of careful electrocardiographic monitoring early during amiodarone therapy to avoid a potentially fatal arrhythmia.
\end{abstract}

Keywords: Amiodarone, Torsades de pointes, QTc interval

\section{INTRODUCTION}

Amiodarone is highly effective for treatment of supraventricular and ventricular tachyarrhythmias. ${ }^{1}$ It has few proarrhythmic effects. ${ }^{2}$ Torsades de pointes (TdP) is an uncommon complication of both oral and intravenous amiodarone. ${ }^{3}$ However, occurrence of $\mathrm{TdP}$ has been observed during chronic amiodarone treatment in conjunction with electrolyte disorders. ${ }^{2,4}$ We describe a case of amiodarone induced recurrent TdP within few days of oral loading in a young boy suffering from dilated cardiomyopathy.

\section{CASE REPORT}

A 16 year old boy presented with complaints of sudden onset palpitations and dizziness in the emergency room. Patient gave history of dyspnea (NYHA class II). He had a blood pressure of 90/64 $\mathrm{mm} \mathrm{Hg}$. ECG revealed ventricular tachycardia (Figure-panel A). Sinus rhythm was restored by $200 \mathrm{~J}$ of synchronized DC shock. His ECG after cardioversion showed a heart rate of 65/minute and $\mathrm{QT}_{\mathrm{c}}$ interval of $435 \mathrm{~ms}$ (Figure-panel B). Echocardiogram showed dilated left and right ventricles with global hypokinesia and ejection fraction of $25 \%$.

\begin{abstract}
Patient was strongly advised implantable cardioverter defibrillator (ICD). While waiting for the same, he was started on metoprolol $25 \mathrm{mg} \mathrm{BD}$ along with oral amiodarone $800 \mathrm{mg} /$ day for 7 days followed by $400 \mathrm{mg}$ OD. On $9^{\text {th }}$ day of initiation of amiodarone, he complained of dizziness and palpitations. The ECG at that time in monitor showed TdP at a rate of 200 beats per minute (Figure- panel C). Sinus rhythm was restored by $300 \mathrm{~J}$ of synchronized DC shock. His serum potassium level was $4.4 \mathrm{mEq} / \mathrm{L}$ and serum magnesium was 1.52 meq/Lt. ECG analysis after restoration of normal sinus rhythm showed significant prolongation of $\mathrm{QT}_{\mathrm{c}}$ interval to $590 \mathrm{~ms}$ (Figure-panel D). ECG's done in the intervening period showed serial $\mathrm{QT}_{\mathrm{c}}$ prolongation. In absence of other precipitating factors and predisposing factors, it was thought that TdP was due to amiodarone induced $\mathrm{QT}_{\mathrm{c}}$ prolongation. The drug was immediately discontinued and beta blockers were initiated. Patient had another episode of torsades and ventricular tachycardia the same day in evening. However, serial ECG's after stopping amiodarone revealed normalization of $\mathrm{QT}_{\mathrm{c}}$ interval. Our patient consented and received (ICD) a week later and has been doing well since then.
\end{abstract}



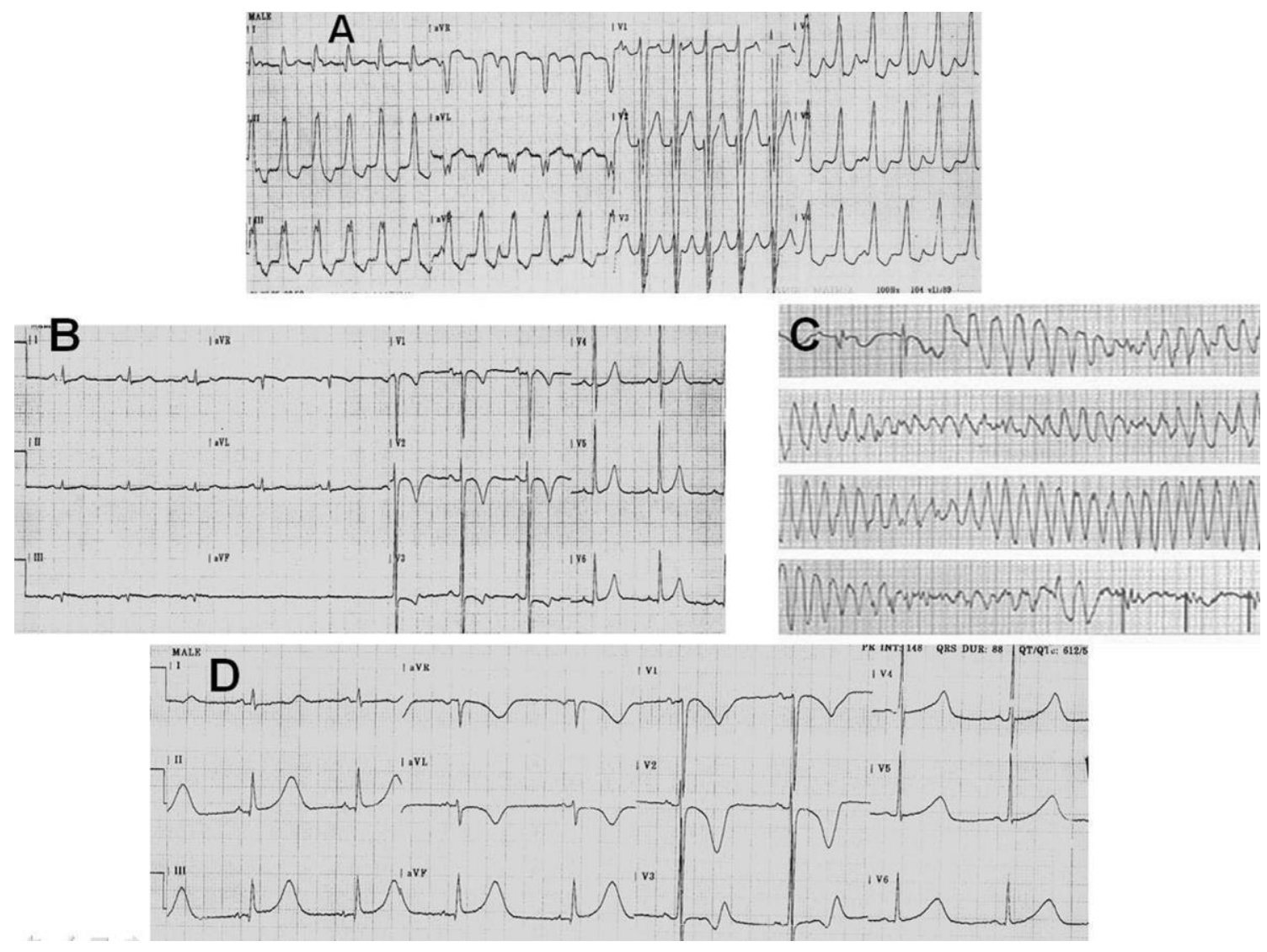

Figure 1: Panel A- ECG showing ventricular tachycardia at the time of presentation; Panel B- ECG in normal sinus rhythm showing a normal QTc interval; Panel C- ECG showing torsades de pointes in patient after starting amiodarone; Panel D- ECG after cardioversion of torsades showing prolonged QTc interval.

\section{DISCUSSION}

Amiodarone increases action potential duration and prolongs refractoriness by blocking delayed rectifier currents. The unique pharmacokinetic properties of amiodarone account for delayed clinical effects but early electrophysiologic effects within 1-2 days. ${ }^{5}$ Overall incidence of TdP due to amiodarone is $0.7 \% .^{2}$ In fact, amiodarone is considered safe even in patients with previous drug mediated torsades. ${ }^{2} \mathrm{TdP}$ in patients on amiodarone develops only in presence of factors prolonging APD like dyselectrolytemias, severe bradycardia, antiarrhythmics and nonantiarrhythmic drugs. ${ }^{6}$ There are several reasons for low incidence of TdP due to amiodarone despite considerable lengthening of QTc and substantial bradycardia. These are lengthening of repolarization ${ }^{2,7}$, abolition of calcium dependent early after depolarizations, shorter prolongation of repolarization in purkinje fibers than in ventricular muscle and due to suppression of preceding ventricular ectopy. ${ }^{8}$ Infact, there is a nonlinear relationship between development of torsades and degree of QT prolongation ${ }^{7}$ with no critical QT duration above which patient is at risk of developing torsades or below which the patient is safe. ${ }^{8}$
The present case deserves attention as our patient developed torsades quite early after administration of oral amiodarone only and that too in absence of any predisposing factors and electrolyte disturbances. This is quite unusual. Furthermore, TdP and ventricular tachycardia recurred highlighting the prolonged QTc interval for some time even after amiodarone discontinuation. After normalization of QTc interval, the arrhythmias didn't recur.

This report highlights the early occurrence of a rare but potentially fatal arrhythmia after amiodarone and emphasizes the need for careful monitoring especially electrocardiographically to avoid any impending fatal arrhythmia.

\section{Funding: None \\ Conflict of Interest: None declared \\ Ethical approval: Not required}

\section{REFERENCES}

1. Podrid PJ. Amiodarone: reevaluation of an old drug. Ann Intern Med 1995;122:689-700. 
2. Hohnloser $\mathrm{SH}$, Klingenheben $\mathrm{T}$, Singh $\mathrm{BN}$ Amiodarone associated proarrhythmic effects. A review with special reference to torsade de pointes tachycardia. Ann Intern Med 1994;121:529-35.

3. Jorens PG, Van Den Heuvel PA, Ranquin REF, Van Den Branden FA, Parizel GA. Amiodarone induced torsades de pointe: Report of three cases and review of literature. Acta Cardiol 1989;5:411-21.

4. Veglia L, Scandiffio T, Guenicchio G. 'Torsioni di punta' e amiodarone. G It Cardiol 1978:8:1025-9.

5. Perry JC. Pharmacologic therapy of arrhythmias. In: Deal BJ, Wolff GS, Gelband H, eds. Arrhythmia in children. Armonk, NY: Futura, 1998:291-3.
6. Breithardt G, Haverkamp W. Concern for the potential for QT prolongation and proarrhythmia by non-antiarrhythmic drugs. Report from a Policy Conference of the European Society of Cardiology. G Ital Cardiol 1999;29(Suppl 5):11-4.

7. Singh BN. When is QT interval antiarrhythmic and when is proarrhythmic? Am J Cardiol 1989;63:8679.

8. Rankin AC, Pringle SD, Cobbe SM. Acute treatment of Torsades de pointes with amiodarone: proarrhythmic and antiarrhythmic association of QT prolongation. Am Heart J 1990;119:185-6.

doi:10.5455/2319-2003.ijbcp20130827

Cite this article as: Gupta MD, MP Girish,

Choudhury PP, Tyagi S. Torsades de pointes in a patient of dilated cardiomyopathy occurring early on oral amiodarone therapy. Int J Basic Clin Pharmacol 2013;2:492-4. 